\title{
Red de docentes de inglés: ¿una posibilidad para la continuidad de grupos de estudio?
}

\author{
An English teachers' network: is it a possibility for \\ continuing study groups?
}

\author{
G. Nathaly Aldana Prieto ${ }^{1}$ \\ Melba Libia Cárdenas ${ }^{2}$
}

Resumen

En la actualidad se promueve la formación de redes de docentes para trabajar en agendas construidas de manera colaborativa, continua, flexible, y conforme a sus necesidades. Reportamos una investigación cualitativa con cinco profesoras de inglés de colegios públicos de Bogotá participantes en un programa de formación permanente. Los profesores conformaron grupos de estudio para realizar un proyecto de investigación-acción. Al concluir dicho programa, las docentes iniciaron un trabajo en red, tendiente a continuar su desarrollo profesional. La información recolectada a través de diarios, cuestionarios y entrevistas, permitió determinar los factores que se asocian a la continuidad de los grupos de estudio que surgieron en el programa de formación permanente, así como algunas implicaciones para el trabajo en red.

\section{Palabras clave}

Grupos de estudio, trabajo en red, desarrollo profesional docente, enseñanza del inglés, investigación en el aula.

Abstract

Nowadays the development of networking on the part of teachers is promoted in order to work with agendas built in a collaborative, continuous, and flexible way, as well as in accordance with teachers' needs. We report on qualitative research of five English teachers in public schools in Bogotá. They took part in a professional development programme and met in study groups in order to conduct an action research project. At the end of the programme the teachers started working as a net with the intent to continue their professional development. Information gathered through journals, surveys, and interviews allowed us to identify factors associated with the continuity of the study groups which emerged in the professional development programme as well as some implications for networking.

\section{Key words}

Study groups, networking, teacher professional development, English language teaching, classroom research.

Artículo recibido el 3 de febrero de 2011 y aprobado el 19 de agosto de 2011

1 Licenciada en Filología e Idiomas-Inglés, de la Universidad Nacional de Colombia, donde fue asistente de investigación de Grupo de Investigación PROFILE (Profesores de Inglés como Lengua Extranjera). Correo electrónico: nathaald@gmail.com

2 Magíster en Teaching English to Speakers of Other Languages de la Universidad de Edimburgo. Profesora asociada de la Universidad Nacional de Colombia, sede Bogotá, y coordinadora del Grupo de Investigación PROFILE (Profesores de Inglés como Lengua Extranjera). Es autora y coordinadora de programas de formación permanente de docentes de inglés y editora de la revista PROFILE. Becaria de la Fundación Carolina, Adelanta estudios de Doctorado en Educación en la Universidad de Zaragoza. Correo electrónico: mlcardenasb@unal.edu.co 


\section{Introducción}

El asunto de la sostenibilidad y continuidad de los procesos adelantados en programas de desarrollo profesional siempre ha sido objeto de preocupación en el área de la formación permanente del profesorado. En Bogotá, contexto donde se adelantó la investigación que nos ocupa, Torres e Isaza (2000) desarrollaron un estudio evaluativo de los Programas de Formación Permanente de Docentes (PFPD) realizados en la ciudad entre 1996 y 2000, y concluyen que, al analizar el impacto de dichos programas, la variable del tiempo juega un papel importante. Por ello recomiendan considerar estrategias para garantizar la sostenibilidad de los PFPD y lograr una mayor incidencia en el ámbito institucional. La Secretaría de Educación de Bogotá (2006) también evaluó los planes territoriales de formación docente y dejó en evidencia el problema de la continuidad de los grupos de trabajo que surgen en los PFPD. En ambos estudios se llama la atención respecto al hecho de que haya grupos que pierden casi por completo el trabajo adelantado durante el transcurso de los mismos y se plantea como solución «acompañar, incentivar la formación de equipos interinstitucionales y colectivos de maestros» (Secretaria de Educación de Bogotá, 2006, p. 25). Esto coincide con los hallazgos de Cárdenas (2004) en lo que respecta a la necesidad de asegurar condiciones para que el camino recorrido en los PFPD no se pierda o se trunque a causa de la falta de nexos debidamente establecidos, una vez ellos finalizan.

A nivel nacional, y de acuerdo con lo que apuntan Cárdenas, González y Álvarez (2010, p. 50), en la última década, y como resultado de los trabajos de investigación y de extensión de diversas universidades, se han ido documentado experiencias que permiten vislumbrar una línea de pensamiento en el área de formación permanente de docentes. Se destacan, en particular, los estudios en torno a las necesidades percibidas por docentes de los sectores público y privado (González, Montoya y Sierra, 2001, 2002), el diseño de un programa holístico para docentes de educación básica primaria (McNulty y Quinchía, 2007), el papel de la investigación-acción en el trabajo de grupos de estudio de docentes de inglés (Cárdenas y Nieto, 2007) y las vivencias de docentes de inglés en la creación de grupos de estudio en un PFPD (Malagón y Cárdenas, 2007; Cárdenas y Nieto, 2010). Asimismo, merecen citarse los trabajos de Álvarez (2009) y Vergara, y de Hernández y Cárdenas (2009), los cuales indagan sobre el conocimiento base de docentes de inglés como lengua extranjera; merece citarse el trabajo de Vergara et al. (2009), que da cuenta de la experiencia de un grupo de profesores universitarios quienes tuvieron a su cargo un seminario de investigación en el aula, dirigido a un grupo de maestros participantes en un programa de desarrollo profesional de la Universidad del Valle.

Desde el año 1995, el Departamento de Lenguas Extranjeras de la Universidad Nacional de Colombia, a través del grupo de investigación PROFILE, ha ofrecido PFPD a los profesores de Bogotá de los sectores público y privado. Desde entonces se ha evidenciado la necesidad de crear redes pedagógicas pues, como advierte Cárdenas (2002), esta clase de comunidades ofrece ventajas en términos de cooperación. No obstante, y a pesar de esfuerzos realizados por docentes de inglés en distintas zonas de la ciudad, no existen trabajos publicados sobre la constitución de redes que permitan el trabajo en equipo, la investigación conjunta y la solución de problemas.

Por lo anterior, nos propusimos indagar sobre la naturaleza del trabajo en red de docentes de inglés, desde sus inicios como grupos de trabajo en un PFPD, y la posibilidad de continuar en contacto luego de finalizar el programa, mediante la constitución de una red (Cárdenas y Nieto, 2010). En esta investigación participó un grupo de docentes de inglés de colegios públicos de Bogotá, así como las tutoras que los acompañaron en el programa en el año 2006 y luego de culminar el programa, en 2007. En el presente artículo nos referimos a los factores que inciden en la continuidad de estos grupos mediante el trabajo en red, para lo cual nos centramos en cinco docentes, quienes nos proporcionaron información a través de diarios, cuestionarios y entrevistas grupales. 
A continuación presentamos el problema de investigación. Seguidamente abordamos el marco conceptual y la metodología. Luego aparecen los resultados basados en las categorías emergentes y finalmente las conclusiones. Entre éstas se encuentra el gran papel que la autonomía de los docentes desempeña en el inicio de este trabajo en red, el acompañamiento de la institución formadora en el transcurso de este proceso y la necesidad del apoyo de las autoridades educativas.

\section{Problema de investigación}

Nos propusimos identificar los factores relacionados con la continuidad de los grupos de estudio que surgen en el marco de un PFPD de inglés, es decir, sus inicios como red de profesores. Para lograr el objetivo principal se plantearon dos objetivos específicos:

- Determinar qué favorece el trabajo en red de profesores de inglés de Bogotá después de su participación en un PFPD.

- Identificar qué circunstancias impiden el desarrollo de dicho trabajo.

\section{Marco teórico}

Tres constructos constituyeron las bases conceptuales que guiaron el estudio que aquí se reporta: los programas de formación permanente de docentes, los grupos de estudio y el trabajo en red.

\section{Programas de Formación Permanente de Docentes (PFPD)}

Concebimos un PFPD como un espacio amplio, como una comunidad académica conformada por profesionales de educación básica, media y universitaria, unida por el interés común de la transformación pedagógica de la escuela y la enseñanza de la lengua inglesa. En el contexto colombiano, los PFPD son ofrecidos a profesores de educación básica y media por universidades, grupos académicos y centros de formación, con el fin de brindar oportunidades para la investigación, la actualización y la innovación. En particular, la Secretaría de Educación de Bogotá (2006) formuló en su Plan territorial de Formación Docente 2006-2007 los lineamientos para futuros PFPD. Se busca que se basen en la relación escuela-ciudad-escuela y continúen siendo impulsadores de una «dinámica académica y de producción pedagógica» (p. 35).

El PFPD PROFILE (Cárdenas, 2006), del Departamento de Lenguas Extranjeras de la Universidad Nacional de Colombia, sede Bogotá, comprende tres componentes que desarrollan la competencia y la enseñanza del inglés: lengua inglesa, metodología e investigación-acción e innovación. Este último se incluye por el valor que la investigación tiene para la universidad y su utilidad en la innovación educativa, ya que, como lo menciona Cárdenas (2004), la investigación en el aula es valiosa si la realizan los actores que hacen parte de ella, máxime si se tiene en cuenta que los profesores son quienes observan $\mathrm{y}$ viven los procesos educativos.

Los profesores participantes en los PFPD tienen la oportunidad de publicar sus investigaciones en la revista PROFILE, que surgió en el 2000 por la necesidad de compartir las experiencias de los docentes. Asimismo, en 2004 tuvo lugar el primer Simposio PROFILE y, desde entonces, los docentes tienen un espacio anual para socializar el proceso y el resultado de sus investigaciones.

\section{Grupos de estudio}

Un grupo de estudio es un colectivo de personas que se reúne voluntariamente para establecer un diálogo, comprender asuntos determinados y llegar a acuerdos teóricos y prácticos (Birchak, Connor, Crawford, Kahn, Kaser, Turner y Short, 1998). Un grupo de estudio crea un sentido de comunidad entre los profesores, que apoya el desarrollo profesional y lo enfoca en transformar la enseñanza a través del diálogo y la reflexión. En palabras de Murphy y Lick (1998), los grupos de estudio buscan incrementar las capacidades de los profesores a través del nuevo aprendizaje y para beneficio de sus estudiantes.

Tres tipos de grupos de estudio, identificados por Birchak et al. (1998), estuvieron presentes en nuestra población objeto: grupos basados en la educación básica y media, grupos centrados en el tema y grupos de investigación. Los grupos basados en la instrucción escolar están conformados por 
educadores dentro de un colegio particular. Los grupos centrados en el tema, reúnen educadores de diferentes colegios, interesados en una misma cuestión. Los grupos de investigación están compuestos por docentes que discuten sus investigaciones en el aula. Ahora bien, adoptando el papel de facilitadores externos, los formadores de profesores sugieren algunas estrategias para la estructura, organización y funciones de los miembros del grupo de estudio. No obstante, las estructuras permanecen flexibles y sin límites fijos.

Oliphant (1998) presenta diferentes factores que se pueden generar en la creación de grupos de profesores. El proceso empezaría con un propósito de soporte emocional o profesional y crecimiento, continuando con la selección de los miembros, el tamaño del grupo, el lugar de los encuentros, las metas generales del grupo, la división de los roles, las actividades para cada reunión, la discusión de posibles problemáticas y la asignación de tareas específicas para cada integrante.

Con base en las conclusiones que surgieron de su propia experiencia de trabajo en grupo, Collay, Dunlap, Enloe y Gagnon (1998) proponen seis condiciones necesarias para que las comunidades de aprendizaje funcionen eficazmente de principio a fin. Nos referimos a las tres condiciones que se relacionan más con los grupos de estudio objetos de esta investigación: construcción de conocimiento, documentación de la reflexión y cambios culturales.

La construcción de conocimiento es vista como un entramado de conocimientos que se van creando, no como imposición de agentes externos, sino como un proceso que hace que cada miembro del grupo analice, viva, descubra y derive sus propias conclusiones. Por su parte, la documentación de la reflexión funciona como una recolección de datos mediante el uso de diarios, la grabación de sesiones del grupo y de clases de cada profesor y el registro de temas y conclusiones en cada sesión. Los registros podrán ser compartidos, lo que permitirá la reflexión permanente que lleva a nuevo conocimiento. Finalmente, los cambios culturales surgen de la exposición a nuevos descubrimientos y cambios sobre el quehacer docente del maestro que hace parte de un círculo de aprendizaje. Sus ideas y pensamientos deben tener un impacto sobre los estudiantes, colegas y su ambiente educativo en general, con lo cual se convierte en un líder y agente de cambio.

En el contexto colombiano, encontramos investigaciones sobre grupos de estudio constituidos por docentes de diversos niveles y sectores educativos. Citamos, en primer lugar, cinco estudios realizados en colegios públicos de Bogotá. Moreno y Palencia (2001) y Ochoa (2004) describen cómo las comunidades de docentes, basadas en el trabajo en la misma institución educativa y mediadas por el apoyo de expertos universitarios, emprenden acciones tendientes a su desarrollo profesional. Ochoa (2005) explora alternativas para apoyar el desarrollo profesional de profesores de primaria no licenciados en inglés y señala implicaciones para las políticas de formación docente. A su vez, Álvarez y Sánchez (2005) indagaron lo que sucedió con un colectivo de profesores de un colegio que participó en un grupo de estudio para acordar un enfoque común para enseñar inglés. Asimismo, Malagón y Cárdenas (2007) identifican las percepciones de grupos de estudio participantes en el PFPD PROFILE respecto al establecimiento de una meta común, las sinergias para avanzar hacia su logro y los aportes para sus prácticas pedagógicas, así como algunas implicaciones para el trabajo colaborativo.

Arias (2008) documentó el trabajo de un grupo de estudio en un colegio privado que buscaba convertirse en institución bilingüe, como una estrategia de desarrollo profesional y de trabajo en equipo para mejorar las prácticas pedagógicas. Por su parte, Castellanos (2009) investigó un grupo de estudio conformado por cinco docentes de cursos de extensión de la Universidad Nacional de Colombia, sede Bogotá. A través de encuentros virtuales, se examinaron las dinámicas para abordar temas consensuados, así como las impresiones de los participantes en relación con su desarrollo profesional.

Encontramos también trabajos realizados sobre grupos de estudio constituidos por formadores de docentes. Sierra (2007a y 2007b) reporta los resultados de una investigación que observó el progreso 
profesional de un grupo de formadores de profesores y futuros profesores de lenguas extranjeras, en una universidad pública en Colombia. El estudio develó los conocimientos, habilidades y actitudes que desarrollaron los profesores como resultado de su participación, así como las habilidades y actitudes que la facilitadora del grupo de estudio desarrolló debido a su papel como observadora participante en el mismo. Se destacan las habilidades de liderazgo, la iniciativa y la actitud democrática. A su vez, el fomento de una propuesta investigativa por parte de algunos docentes de lenguas de un grupo de estudio en autonomía y nuevas tecnologías, permitió confirmar la pertinencia de dicho sistema de trabajo para el desarrollo profesional, e identificar rasgos que debería poseer una persona que decide pertenecer a un grupo de estudio: actitudes, habilidades, valores y conocimientos (Gómez y Pineda, 2009).

\section{Trabajo en red}

Torres e Isaza (2000), en su estudio sobre la evaluación de los PFPD realizados en Bogotá entre 1996 y 2000, aseguran que se deben tener en cuenta la conformación de grupos y el seguimiento a los participantes y sus instituciones. Además, en la evaluación de los planes territoriales 2004 y 2005 se encontraron varios desaciertos, entre los que se destacan la desarticulación en las acciones realizadas (Secretaría de Educación de Bogotá, 2006) debido a la no continuidad de los grupos de trabajo. Con el fin de superar esta situación se propone como objetivo para los subsiguientes PFPD «acompañar e incentivar la formación de equipos interinstitucionales y colectivos de maestros, interesados en ampliar y profundizar el estudio de temas y asuntos relacionados con su labor profesional» (p. 25).

Una de las nuevas propuestas para la formación docente es la organización de los maestros en redes, por cuanto permiten construir una comunidad de puesta en común y de apoyo de grupo (Bojacá, 2001). Martínez Pineda (2004) define las redes como grupos de profesores que se reúnen para los programas de formación permanente y para analizar su papel en la sociedad. Calvo (2004), en su estudio sobre la formación de los docentes en
Colombia, precisa que en esta clase de organización los maestros se encargan de «realizar procesos de formación profesional para reflexionar sobre su rol, sus alcances, sus realidades, a fin de interpretarlas y reconstruirlas» (p. 135). La autora concluye que las redes surgen gracias a una necesidad de estudiar y formarse con personas que sí conocen la realidad y el contexto de la educación en Colombia. Por su parte, la Secretaría de Educación de Bogotá (2008) señala que una red puede concebirse como

el asocio de grupos de estudio, trabajo o investigación -colectivos de docentes en este caso-, maestros y maestras con intereses comunes para el desarrollo de saberes transversales y específicos, apoyados por metodologías disciplinares e interdisciplinares, pero que, fundamentalmente, la red constituida por dichos colectivos busca-entre otros- legitimar espacios, legitimar conocimientos, legitimar la trayectoria que puede tener como colectivo alrededor de un tema de interés común que los convoque (p. 4).

En nuestro trabajo en diversos PFPD hemos observado la importancia de la construcción de redes de profesores de inglés. Como señala Cárdenas (2002), existen múltiples ventajas de las comunidades de profesores en la investigación, a saber: el trabajo en equipo, la cooperación y retroalimentación, la posibilidad de llevar a la práctica la teoría y de ser más perceptivos y mejor observadores en sus clases. Además, muchos docentes participantes en PFPD reconocen la necesidad de trabajar en grupo para compartir información, aclarar dudas y apoyarse en los procesos educativos (Cárdenas, 2004).

Debido a la ausencia de una red de profesores de inglés en Bogotá se propuso el PFPD Red PROFILE: Red de profesores de inglés como lengua extranjera para el fortalecimiento de sus competencias comunicativas, pedagógicas e investigativas en la educación básica y media (Cárdenas, 2006). El programa buscó inicialmente la constitución de grupos de estudio para adelantar proyectos de investigación, lo cual implicó el trabajo en red entre docentes de una misma o diversas instituciones y formadores de formadores, en calidad de tutores. Se buscaba también motivar redes interinstitucionales que inte- 
ractuarían después con otras de su misma localidad o de diferentes zonas de Bogotá.

El PFPD en mención parte de la definición de una red como una reunión de docentes de educación básica y media y de profesores-formadores, unidos por la voluntad explícita de estudiar, examinar y resolver fenómenos y problemas pedagógicos, así como por el interés por la cualificación de la pedagogía de la lengua inglesa. Puesto que en Colombia no tenemos mucha experiencia en esta clase de comunidades en el área de inglés, Cárdenas (2002) propone que profesores junto a formadores de formadores reflexionen e investiguen en contextos a los que pertenezcan, permitiendo la creación de grupos de estudio que continúen su trabajo, sin necesidad de agentes externos. Estos grupos de estudio se generan ya sea porque los profesores pertenecen al mismo colegio, porque comparten tendencias respecto a un tema o porque están interesados en resolver dudas o inquietudes a través de la investigación.

Pasar de trabajar en un grupo de estudio a hacerlo en una red es, de acuerdo con Kirschner, Dickinson y Blosser (1996), pasar de hacer un trabajo cooperativo a uno colaborativo. En el primero, los miembros del grupo se reúnen para lograr un objetivo, mientras que en el segundo se requiere inquietud intelectual conjunta (p. 206). Así, el enriquecimiento será recíproco, pues el trabajo en red propicia el trabajo colaborativo. Su dinámica favorece procesos de aprendizaje, la circulación de conocimientos, la puesta en común de experiencias y conocimientos significativos y aplicaciones locales.

\section{Metodología}

Se realizó un estudio de caso cualitativo siguiendo el modelo de Strauss y Corbin (1990), quienes califican la investigación cualitativa como «el proceso no matemático de interpretación [...] con el propósito de descubrir conceptos y relaciones» (p. 12). En particular, realizamos un estudio de caso de tipo interpretativo. Siguiendo los postulados de Brown (2002) y Merriam (1988), nos enfocamos en un grupo de personas pertenecientes a una comunidad determinada, en circunstancias establecidas, lo cual nos permitió realizar una descripción detallada de los participantes y el objeto de estudio.

En cuanto a la categorización de los datos, se realizó de acuerdo con la teoría fundamentada que presentan Corbin y Strauss (1990), donde la recolección de datos, el análisis y la teoría guardan estrecha relación entre sí.

\section{Participantes}

Tomamos como población objeto el PFPD Red PROFILE, que se desarrolló en 2006 y fue auspiciado por la Secretaría de Educación de Bogotá. El programa benefició a 40 docentes de colegios públicos, quienes estudiaron los sábados, entre las 7 a.m. y las $12 \mathrm{~m}$., alrededor de tres componentes: lengua inglesa, metodología, e investigaciónacción e innovación. En este último módulo, los docentes fueron orientados en la realización de un proyecto en sus colegios, para lo cual se asociaron en 13 grupos de estudio. Al finalizar el programa se planteó la posibilidad de continuar el trabajo adelantado en el grupo, en forma de red. Para ello se constituyeron espacios para una red de docentes de inglés, no sujeta a los requerimientos del PFPD: comunicación por vía telefónica y electrónica, acuerdos de temas y sistemas de trabajo, encuentros con docentes y auxiliares de investigación para abordar asuntos de interés común -liderados por tres tutoras- con hablantes nativos invitados o con los mismos docentes.

Las reuniones de la red de profesores de inglés se realizaron un sábado cada mes durante el 2007, entre las 8 a.m. y las $12 \mathrm{~m}$. en las instalaciones de la Universidad Nacional de Colombia. Las fechas de los encuentros, así como los temas planteados, eran acordadas por los docentes. De esta manera la metodología, sus habilidades del lenguaje y vivencias, son los temas que se trataban con más frecuencia.

Las participantes en el presente estudio fueron 5 profesoras, quienes asistieron a las reuniones de la red por lo menos en dos ocasiones y firmaron un consentimiento informado, preparado por el Grupo de Investigación PROFILE para su proyecto macro. En él registraron el nombre con el cual deseaban ser 
llamadas en las publicaciones: Catalina, Mariana, Alondra, Cristina y Adriana.

Catalina y Mariana son licenciadas en lenguas modernas y ambas poseen títulos de especialización; se desempeñan como profesoras de inglés y español en la jornada de la mañana. Catalina enseña 15 horas semanales en los grados $6^{\circ}, 8^{\circ}$ y $10^{\circ}$ y Mariana 2 horas semanales en $6^{\circ}$. No tenían experiencia en investigación y tampoco pertenecían a una red o grupo de estudio. Cristina trabaja en la jornada tarde. Enseña inglés 21 horas a la semana en los grados $6^{\circ}, 7^{\circ}$ y $10^{\circ}$ y también se desempeña como profesora de ética. Es licenciada en lenguas modernas; obtuvo un postgrado y manifiesta pertenecer a la red de Bilingüismo apoyada por la Secretaría de Educación de Bogotá 3 . Por su parte, Adriana es licenciada en lenguas modernas con una especialización y labora durante 15 horas, en la jornada tarde, en el grado 11. Vivió sus primeras experiencias en investigación y en trabajo en red en el PFPD. A su vez, Alondra es licenciada en lenguas modernas y labora 20 horas a la semana, en la jornada de la mañana, como profesora de español e inglés de $7^{\circ}$ a $10^{\circ}$. Al ingresar al PFPD ya poseía experiencia en investigación e innovación en proyectos colectivos en su institución educativa. Finalmente, y en relación con el escalafón docente que las rige, encontramos que Catalina, Mariana y Adriana pertenecían al régimen establecido por el Decreto 2277 (Ministerio de Educación, 1979), mientras que Cristina y Alondra, al Decreto 1278 (Ministerio de Educación, 2002).

\section{Proceso de recolección de datos}

El estudio se adelantó en cuatro etapas. En la primera se seleccionó la muestra y en la segunda se identificaron las percepciones de los docentes en torno a la posibilidad de trabajar en red, luego de participar en grupos de estudio. En la tercera etapa, se buscó identificar los diferentes factores que se relacionan con el inicio de un trabajo en red de los profesores después del PFPD. Finalmente, en la cuarta etapa se analizaron los rasgos distintivos que

3 No se conocen documentos reglamentarios o productos de la misma. llevan a los grupos a ampliar su horizonte mediante el trabajo en red.

Los datos se recogieron durante y después del PFPD a través de diarios, entrevistas y cuestionarios. Los profesores llevaron un diario durante el transcurso del PFPD donde plasmaban sus experiencias y vivencias en el transcurso del programa, al trabajar en equipo o al realizar su proyecto de innovación o investigación, así como sus percepciones hacia una posible continuidad (ver ejemplo Figura 1). Para efectos de este estudio, tomamos como primera fuente de análisis la última entrada del diario. Sus reflexiones nos permitieron indagar sobre la posibilidad del trabajo en grupo adelantado durante el PFPD. Es de anotar que Cristina fue la única participante que no escribió la última entrada del diario.

Figura 1. Ejemplo de un diario de campo. Último registro del diario de Alondra

December 11, 2006

I'm not going to work with my study group because we are at different schools and besides we have had many problems, especially with Andrea, because she is an incomprehensible person and she doesn't like to work in group.

However, I would like to continue working with Cristina. She is a special person. She helped me, and we worked very hard. Besides, I learned many interesting things for my professional life. I would like to work in this research for a long time and apply the lesson plans to other levels and implement it in Christina's school so that we can see what happens with these students... and I can get more results.

Optamos por la entrevista estructurada y de carácter grupal, para lo cual se siguió un orden de preguntas previamente establecido, que nos permitiera centrarnos en ciertos aspectos y plantear otros interrogantes, de tal forma que las participantes pudieran ahondar en los temas propuestos. Esto dio lugar a pequeñas discusiones y a que se reforzaran ideas presentadas por las profesoras.

Se realizaron dos entrevistas, en distintos momentos y de manera simultánea, por diferentes miembros del Grupo de Investigación PROFILE. La primera tuvo lugar al culminar la reunión inicial sostenida luego del PFPD y como una forma de dar continuidad al trabajo adelantado en él. El objetivo de esta entrevista fue ahondar en los factores relacionados con el trabajo en red (ver Figura 2). 
Figura 2. Protocolo usado para la primera entrevista

1. ¿Cómo se sintió como miembro del grupo durante el desarrollo del proyecto?

2. ¿Qué acciones llevó a cabo para incrementar/motivar/incentivar el trabajo en grupo?

3. ¿Sigue en contacto con los miembros del grupo?

4. Si la respuesta es afirmativa, ¿de qué manera?

5. Si la respuesta es negativa ¿por qué no?

6. ¿Qué papel han jugado sus compañeros del área de inglés en la continuidad o no de su trabajo?

7. ¿Se han involucrado?

8. ¿Se interesan?

9. Si nos ubicamos en el contexto institucional, ¿qué ha apoyado o impedido, desde las directivas de su institución, la continuidad del trabajo en su grupo?

La segunda entrevista se realizó después de 6 meses de haber comenzado las sesiones periódicas de la red. Además de hablar sobre el trabajo en red, la entrevista buscó resaltar las posibles estrategias para facilitar dicho trabajo (ver Figura 3).
Figura 3. Protocolo usado para la segunda entrevista

1. ¿Qué se debe hacer en una red como esta?

2. ¿Qué utilidad encuentra en ese trabajo?

3. ¿Qué circunstancias o factores se requieren para que pueda vincularse a la red?

4. ¿Ha asistido a encuentros?

5. ¿Qué le permitió asistir?

6. ¿Qué lo motivó a asistir?

7. ¿Hay algo que le haya impedido o que de alguna forma le haya hecho más difícil asistir a los encuentros?

8. ¿Qué ha hecho para sobrepasar esas dificultades?

9. ¿Qué circunstancias o factores han favorecido su trabajo en red?

10. ¿De qué manera mejoraría el trabajo en red que se ha hecho hasta el momento?

11. ¿Qué estrategias pueden fomentar ese trabajo en red?

Por su parte, el cuestionario se empleó al finalizar el PFPD, con el fin de identificar percepciones de las profesoras sobre el trabajo en red, en términos de favorabilidad, impedimentos y acciones para sobrepasarlos (ver Figura 4).

Figura 4. Cuestionario empleado al finalizar el PFPD

Apreciado(a) Profesor(a):

El presente cuestionario busca recoger información sobre alternativas de sostenibilidad de lo adelantado en el presente PFPD.

Si lo considera necesario, amplíe sus respuestas en una hoja adicional.

Nombre del (la) docente: Cristina

ALTERNATIVAS PARA EL TRABAJO EN RED:

A. Posibilidades para continuar desarrollando en su institución, en el año 2006, el proyecto consolidado en este PFPD Creo que podría ser considerado por los profesores de la asignatura para dar viabilidad a su aplicación en la programación.

B. Condiciones requeridas para hacer posible esa alternativa

Divulgación del proyecto, recursos.

C. Probabilidad para el establecimiento de redes de trabajo de docentes de inglés Subraye la opción correspondiente:

1. En su institución: Muy probable Probable Poco probable

Porque siendo una institución pequeña, podría darse esta posibilidad, aunque generalmente la gran dificultad son los tiempos.

2. En su localidad: Muy probable Probable Poco probable

Porque el gerente del Cadel siempre está dispuesto a apoyar iniciativas que redunden en beneficio de la comunidad educativa.

3. A nivel distrital: Muy probable Probable Poco probable

Porque no se da el tiempo requerido para este tipo de ejercicio. Y es difícil que los profesores brinden tiempo extra para este trabajo.

D. Sugerencias o requisitos para el trabajo en red en su institución

Separar las reuniones del área español-inglés con el fin de hacerlas más específicas y optimizar el tiempo.

E. Sugerencias para el trabajo en red en su localidad

Dar algún tipo de motivación a quienes hagan parte de esta red.

F. Sugerencias/requisitos para el trabajo en red a nivel distrital

Primer requisito, que todos los docentes de inglés hagan el PFPD y tengan la visión y el interés de investigar y profundizar.

OTROS COMENTARIOS: 
Finalmente, es preciso señalar que nuestra participación en la investigación se dio gracias al trabajo de las autoras como auxiliar del grupo de investigación (Nathaly) e investigadora principal (Melba Libia). La primera se desempeñó como observadora y la segunda, como observadora-participante, por cuanto fue autora y tutora del programa.

\section{Resultados}

Para el estudio de los datos tomamos como unidades de análisis los enunciados, donde las profesoras expresaban sus opiniones respecto a las circunstancias, actores o situaciones relacionados con el trabajo en red y la continuidad de los grupos de estudio después del PFPD. A su vez, y siguiendo los postulados de Strauss y Corbin (1990), se realizó una codificación abierta.

La validez de la información se aseguró a través de dos de las cinco formas propuestas por Burns (1999, p.p. 163-164): triangulación por métodos y triangulación por tiempo. Según la misma autora, la triangulación nos permite hacer el estudio teniendo en cuenta diferentes perspectivas. La triangulación por métodos -o instrumentos de recolección de datos- permitió encontrar la misma información a través de los 3 instrumentos utilizados, y la trian- gulación por tiempo permitió, a su vez, dar cuenta del proceso de los profesores en tres momentos diferentes (al finalizar el PFPD, al comenzar y al culminar las reuniones de la red).

Los diarios, las entrevistas y los cuestionarios se analizaron por separado. El análisis de los diarios y de la primera entrevista permitió identificar los factores que impiden o permiten el trabajo en red. La segunda entrevista y los cuestionarios, además de mostrar las dificultades y aspectos que favorecen el funcionamiento del trabajo, dejaron en evidencia estrategias para contrarrestar esas dificultades y mejorar el trabajo en red.

El análisis permitió encontrar códigos que se repetían en los diferentes instrumentos. Es así como se agruparon los códigos que se relacionaban, teniendo en cuenta la frecuencia con que aparecían en los documentos, y se identificaron las categorías y subcategorías. Como puede observarse en la Gráfica 1 , estas dan cuenta de los factores relacionados con la continuidad de los grupos de estudio que surgen en el PFPD, hacia los inicios de un trabajo en red. Asimismo, las 3 categorías y las sub-categorías que se derivan de ellas nos permiten establecer qué favorece y qué impide la continuidad del trabajo en red de los profesores.

Gráfica 1. Categorías y sub-categorías emergentes del análisis de datos.

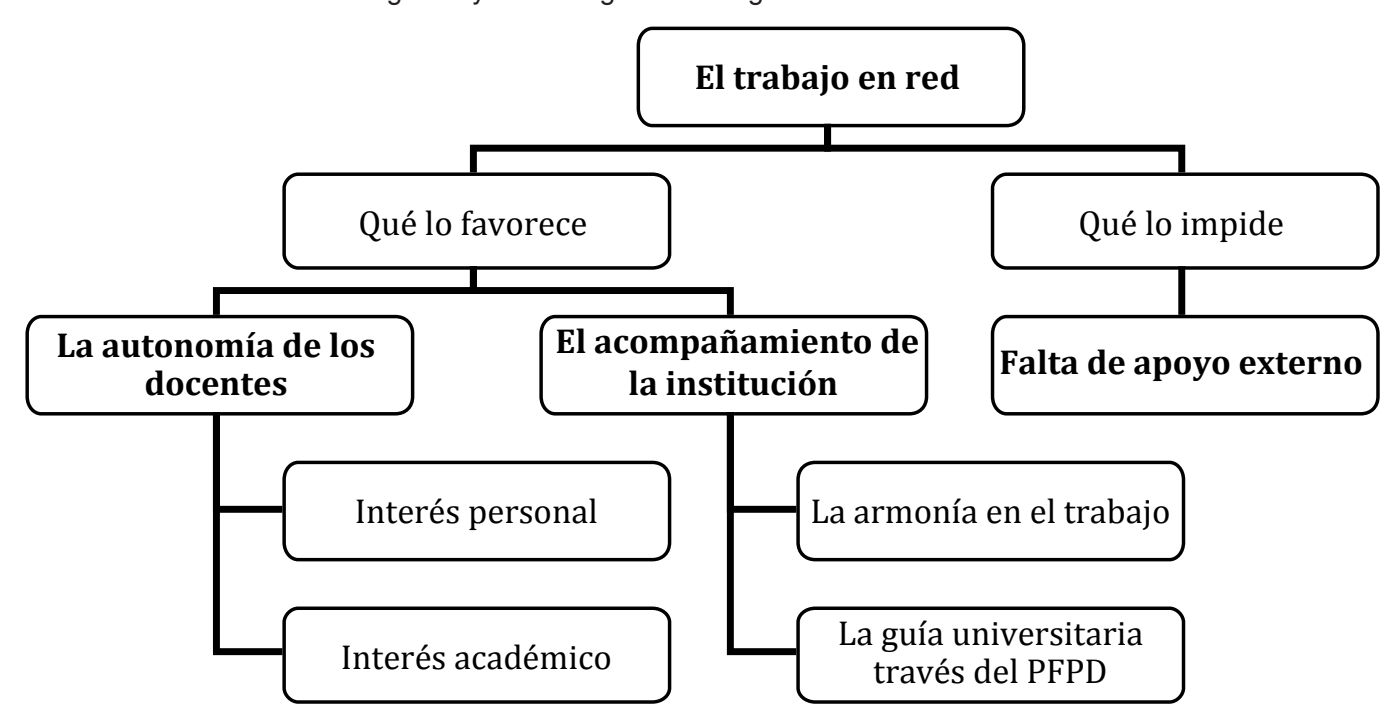




\section{La autonomía de los docentes como base del trabajo en red}

Las situaciones que afrontan los docentes en sus sitios de trabajo hacen su labor cada vez más difícil. En muchos casos se enfrentan a tensiones con compañeros debido a la diferencia generacional o al desinterés en expandirse a otras áreas, lo que se convierte en un impedimento a la hora de hacer proyectos comunes. Además, los colegios distritales ${ }^{4}$ consideran que el desarrollo de proyectos es vital, pero esto también implica labores adicionales para el profesor en ejercicio. No obstante, las directivas de las instituciones no ofrecen el apoyo suficiente para que las ideas de los profesores se ejecuten y den los frutos esperados. Todo esto, unido al agotamiento, al exceso de trabajo y a los compromisos familiares, nos llevó a preguntarnos qué hace que el docente investigue sobre su quehacer y asista a los encuentros en red.

Por tantas actividades, como uno en el colegio desarrolla tantos proyectos, decimos a veces la 'proyectitis'. Son proyectos de una cosa, de otra... Entonces como que uno tiene que cumplir con tantas cosas en el colegio, que uno va dejando de lado eso que puede ser... (Entrevista 1, Cristina)

El vincularse y trabajar en una posible red de profesores de inglés promovida por el PFPD no es una labor obligatoria para los docentes del Distrito. Encontramos que los miembros de la red participan en esta, en primera instancia, por motivaciones personales y profesionales.

\section{Interés personal}

Tiene que ver con el compromiso, con la responsabilidad que cada uno tenemos, con cómo podemos dejar a veces la pereza de lado para venir y asistir y decir... Bueno, yo quiero, me interesa esto, esto es lo que... por aquí es que voy bien encaminada. Y no voy a ganar más con esto monetariamente... pero sí estoy mejorando mi calidad profesional y personal. (Entrevista 2, Catalina)

4 Término con el cual también se denominan los colegios públicos de Bogotá.
Las palabras de Catalina evidencian el papel preponderante de la autonomía en el trabajo que se hace en una red. Si bien es cierto que la red funciona como un enlace entre docentes de distintas localidades y que es una oportunidad para intercambiar experiencias, el primer impulso que los miembros tienen para participar en ella viene de su interior: del interés personal (como ellos mismos lo llaman) que los motiva a dedicar tiempo al ejercicio en red. Ese interés personal hace alusión a mejorar, entre otros aspectos, sus competencias en la lengua inglesa, como indica una docente: «Además, para mejorar nuestro nivel de inglés [...] la parte personal también es muy útil» (Entrevista 2, Mariana).

La motivación que los profesores tenían antes de involucrarse a la red se presenta como un factor que los impulsa y que, con el paso del tiempo, impulsa a otros a vincularse. Si la motivación es lo suficientemente fuerte, se convierte en responsabilidad; la misma que manifiestan sentir hacia su labor docente. Observamos que debido a la naturaleza de la red, en donde el trabajo se presenta de manera horizontal, cada miembro actúa como un eslabón y cumple una función específica que a lo largo del tiempo permitirá la construcción de una cadena fuerte y sólida. Es por esto que desde la misma vinculación a la red los docentes adquieren una responsabilidad consigo mismos y con los demás miembros, como indican los siguientes testimonios:

\footnotetext{
Porque hay muchos docentes de inglés que ya han tomado este PFPD y han quedado muy motivados para seguir en contacto con esto. (Diario, Catalina)

Pienso que lo más importante es la actitud de los docentes ante esta propuesta. Cuando la gente quiere involucrarse, se hace más fácil conseguir este objetivo. (Cuestionario, Mariana)
}

Una vez se han motivado para participar en la red y se han involucrado efectivamente en ella, las docentes, como parte vital del grupo, adquieren un compromiso personal con el crecimiento y evolución de la misma. Entonces buscan acciones que salgan de su propia experiencia para fortalecerla y darla a conocer, pues es de gran motivación escuchar 
sobre un proceso de crecimiento personal, gracias a una labor en equipo. Es así como algunas de ellas se interesan por comunicarse con pares académicos para invitarlos a ampliar el horizonte de la red.

Creo que de pronto también uno a veces, yo no me comunico con mucha gente diferente del PFPD pero podría ser una estrategia uno poderse comunicar y decirle 'oye, mira... esto ha pasado, está pasando. Es interesante, es muy interesante... ¿por qué no vas, miras a ver cómo está, cómo se están desarrollando allá las reuniones. (Entrevista 2, Catalina)

Conozco una persona que entró ahoritica al Distrito pero no es especializada en inglés pero trabajó unos años en inglés y estudió una parte de su carrera... Hizo como seis semestres de inglés... y después estudió preescolar. Y yo sé que por ejemplo esa persona estaría muy interesada en hacer parte de este grupo. O sea, sería como mirar si se puede ampliar la participación de otras personas que así de pronto no cumplan con ciertos requisitos que se han establecido desde antes... están deseosas de participar... puede enriquecer esa parte del proyecto. (Entrevista 2, Adriana)

\section{Interés académico}

Los docentes tienen siempre la necesidad de trascender profesionalmente, y el trabajo en red les ofrece la oportunidad de abordar las inquietudes académicas que surgen diariamente en su profesión. En nuestro caso, el trabajo mancomunado a lo largo del programa de formación permanente y los encuentros posteriores de la red, mostraron que los intereses académicos de los docentes se centraron en tres áreas: en la investigación, la divulgación de la misma y en asistir a eventos para mantenerse actualizados.

La participación en el PFPD sensibilizó a los docentes hacia la investigación en educación. Como puede constatarse en los siguientes testimonios, el nuevo campo les permite solucionar problemas de sus estudiantes, enfrentar inconvenientes en su labor docente y planear mejoras educativas en su institución. El trabajo en red es el espacio que tienen para llevar sus inquietudes o propuestas, organizarse en torno al tema, recibir retroalimentación y, teniendo un propósito común que los une, sacar adelante la investigación.

Me gustaría trabajar en esta investigación por un buen tiempo y poner en práctica las planeaciones de clase en otros niveles, en el colegio de Cristina, para observar qué ocurre con estos estudiantes. Así puedo obtener más resultados. [I would like to work in this research for a long time and apply the lesson plans to other levels and implement it in Christina's school to see what happens with these students. This way, I can get more results] (Diario, Alondra).

[...] el impulso que siempre se está dando a que retomemos nuestro rol de investigadores, porque nosotros somos investigadores innatos pero necesitamos organizarnos más, necesitamos proyectarnos más (Entrevista 2, Adriana).

El Grupo de Investigación PROFILE motiva a los docentes del PFPD a publicar los resultados de sus investigaciones en la revista que lidera ${ }^{5}$. Los profesores reconocen que esta es una oportunidad muy especial porque a través de la publicación de su trabajo se puede evidenciar la importancia del mismo, se comparte la experiencia y se aporta a un grupo más grande de docentes a nivel nacional e internacional.

[...] tratar de escribir más eh... publicar... que eso también va a ser muy beneficioso para nosotros (Entrevista 2, Adriana)

La idea es empezar a escribir el 'Scholarly research report'. Además, hemos pensado que sería interesante seguir investigando en el tema que escogimos pues como trabajamos con los niños de sexto se podría seguir ahondando en la técnica de 'skimming' y explorar otras técnicas para mejorar la comprensión de lectura. (Diario, Mariana)

5 La revista PROFILE, Issues in Teacher's Professional Development es una publicación semestral del Departamento de Lenguas Extranjeras de La Universidad Nacional de Colombia. Promueve, en primera instancia, la divulgación de trabajos de investigación en el aula -de lengua inglesa- de profesores de educación básica y media. Divulga también investigaciones, innovaciones y reflexiones de formadores de docentes y de profesores de distintos niveles educativos. 
Cárdenas (2003) hace alusión a una investigación sobre las experiencias que vivieron algunos profesores como investigadores y escritores en un PFPD y hace énfasis en la necesidad de la práctica de la escritura de los profesores en ejercicio para compartir sus vivencias e innovaciones. Cabe señalar que durante el PFPD se brindan directrices para la escritura académica y se exige una producción concreta, en forma de un reporte de investigación. Posteriormente, se anima a los profesores a presentarlo para evaluación en dicha publicación, conforme a los lineamientos establecidos para ello. A su vez, a partir de la necesidad sentida por los docentes, la mecánica de los encuentros de la red les brindó herramientas para desarrollar habilidades útiles a la hora de escribir artículos, hecho que se constituye en una de las utilidades principales que encontraron en las reuniones de la red.

Cada sesión de la red se planeó de acuerdo con las necesidades e inquietudes que presentaban los docentes. Esto fue otra fuente de motivación para la continuación en ella pues los docentes se encontraron nuevamente y se dieron respuestas y ayuda mutua respecto a sus dudas. El tema que más interesa tratar en los encuentros es sin duda alguna la metodología. Los docentes se mostraron deseosos de ampliar su conocimiento sobre el tema y aplicar nuevas estrategias. En este punto era muy importante para ellos hablar de sus experiencias, de lo que pasaba en el aula y encontrar conjuntamente una salida viable a las dificultades.

Como profesional creo que me aporta en gran medida para mi, para mi, para mi mejoramiento en el inglés y para mi desarrollo en clase con los estudiantes porque aprende uno cosas acá. No necesita tantos recursos para utilizar en la clase... Aunque los recursos son escasos... una actividad, por ejemplo, la de la pasada sesión... con eso innova uno en la clase y les interesa a los estudiantes. O sea aporta muchísimo. (Entrevista 2, Cristina)

En esta red se adquieren muchos conocimientos a nivel gramatical, a nivel de habla, también a nivel de las vivencias que tiene cada uno de los docentes (Entrevista 2, Alondra).
Otro interés académico que se evidenció en este estudio fue el de mantenerse en contacto con la Universidad. Para ellos era muy importante estar al tanto de los eventos académicos en los que podían involucrarse como ponentes o participantes, ya que es otra forma de actualización. Es aquí donde la red funciona como vía de comunicación entre docentes, puesto que aunque están en la misma ciudad, no tienen acceso a información sobre los mismos.

De los eventos que se realizan acá, que son compartir experiencias exitosas, experiencias de diferentes personas que están interesadas en ese tema. Entonces a mí me parece que una red es bien importante porque no está uno como allá aislado, sino que está al tanto de todas las cosas que pasan, de los eventos, de cosas nuevas. (Entrevista 2, Cristina)

Yo creo que otro aspecto es mantenernos actualizados...Entonces cuando uno está ahí... se entera de eventos... A veces uno está por allá desconectado con sus compañeros que ni idea, no sabe... Es mantenerse actualizado. (Entrevista 2, Catalina)

\section{El acompañamiento de la institución formadora}

Como ya se mencionó, estudios adelantados sobre PFPD ofrecidos en Bogotá (Torres e Izaza, 2000; Secretaría de Educación de Bogotá, 2006), así como en el marco del PFPD en el que se desarrolló esta investigación (Cárdenas, 2004; Cárdenas y Nieto, 2007; Malagón y Cárdenas, 2007), muestran la preocupación por la falta de continuidad del trabajo adelantado en dichos programas. En consecuencia, el Grupo de Investigación PROFILE decidió promover la constitución de una red de docentes de inglés, constituida principalmente por profesores que realizaron el PFPD en el año 2006. Se decidió emprender un acompañamiento permanente a los miembros de dicha red $y$, por sugerencia de los mismos docentes, acoger en los encuentros a todos los docentes de inglés que quisieran participar en ellos.

La labor continua del docente y la Universidad creó un vínculo en donde el trabajo no era visto como una obligación, sino como un espacio ameno para compartir. El análisis de lo observado en los 
encuentros y de lo expresado por las docentes participantes en nuestro estudio, nos permite explicar cómo perciben el trabajo armónico y la guía que reciben del PFPD, y por ende de la Universidad.

\section{La armonía en el trabajo}

Las docentes manifestaron la importancia de tener un ambiente agradable cuando se trabaja en grupo. Después de una semana de trabajo y de tensiones en los colegios, las docentes buscaban un espacio donde no existieran conflictos. En poco tiempo encontraron el ambiente de la red acogedor y perfecto para desestresarse. La conexión universidad-docentes se presenta en la red sin ninguna clase de protocolos, expresando de manera sincera las circunstancias que posibilitan o dificultan su asistencia a los encuentros.

Hubo como circunstancias personales ¿no?, como personales y familiares que me impidieron asistir y hubo momentos en que yo también estaba como... No puedo negarlo: este año ha sido como difícil. Entonces también ha sido como una desmotivación desde lo personal... Hoy me doy cuenta que es un espacio donde uno puede compartir y distraerse un poco de sus otras preocupaciones y es un espacio agradable. Lo que decía Mariana. Sí, como la calidez, como que lo tengan en cuenta a uno... Eso es importante para que uno se motive a continuar. (Entrevista 2, Catalina)

La acogida que hemos tenido por parte del grupo PROFILE, o sea, el trabajo que se ha hecho o las personas que están dirigiendo el trabajo, pues lo hacen de una manera muy profesional y muy cálida también. Entonces el trabajo se vuelve más ameno, más agradable y uno va aprendiendo a la vez que va disfrutando de sus encuentros. (Entrevista 2, Mariana)

Observamos el papel fundamental que juega la Universidad, como institución formadora, en la constitución y avance de la red. Los docentes reconocen el ambiente de trabajo, la guía ofrecida, la puntualidad de los eventos, la planeación oportuna de las actividades, el cumplimiento de las agendas acordadas y el simple hecho de compartir un café.
Todo esto creó armonía y generó una atmósfera diferente a la del colegio.

Por otra parte, la compatibilidad docentedocente es fundamental en el trabajo en red, en la medida en que genera armonía en las labores, lo que a su vez agiliza las actividades y permite crear confianza. Observamos que los docentes asistentes a los encuentros compartían la misma realidad, se identificaban el uno con el otro y creaban lazos cada vez más estrechos. Sin embargo, también hallamos preferencias entre algunas participantes en el estudio, pues nos indican el interés por continuar trabajando con colegas por quienes manifiestan afinidades afectivas o porque adelantaron conjuntamente su proyecto o trabajos previos.

Sin embargo, me gustaría continuar trabajando con Nina. Ella es una persona especial. Me ayudó y trabajamos arduamente. (Diario, Alondra. Escrito originalmente en inglés. La traducción es nuestra)

Deseo trabajar con mis dos compañeras, María y Mariana... Han sido compañeras maravillosas... Por esta razón deseo, de ser posible, trabajar con mis dos compañeras... Trabajamos bien. (Diario, Catalina. Escrito originalmente en inglés. La traducción es nuestra)

Una de las razones de ser de la red de docentes de inglés, es la posibilidad de dar a conocer sus experiencias, lo que vivían en las aulas, y a su vez, escuchar las vivencias de los demás para aprender mutuamente y buscar alternativas enriquecedoras. Esto es posible gracias a que tienen y comparten metas profesionales y personales, como nos muestran estas profesoras:

No sólo nos quedamos en el aula sino que tenemos la oportunidad de, primero de compartir experiencias con otras personas o con otros docentes y segundo, aprender de esos docentes de sus, de cada uno de sus proyectos, de cada una de sus investigaciones. (Entrevista 2, Alondra)

Como de cambiar de ambiente, de venir a una institución donde la academia está presente. El ambiente es diferente... Y donde uno se siente acompañado también de otras personas con las 
que se conoció antes, pero está estableciendo unos lazos más estrechos. (Entrevista 2, Adriana)

Lo anterior coincide con los resultados del estudio adelantado por Sierra (2007a, 2007b), al examinar el devenir de un grupo de estudio. Observamos que en una comunidad de aprendizaje es crucial tener una atmósfera cómoda y libre de tensiones, pues así se permite a los docentes expresar con libertad sus puntos de vista, hacer preguntas sin sentirse intimidados, ampliar sus conocimientos a través de discusiones y experiencias compartidas y crear un espacio de aprendizaje mutuo.

\section{La guía universitaria a través del PFPD}

Como se mencionó anteriormente, la presencia de la Universidad a través del grupo de investigación que lidera el PFPD fue vital para el inicio y funcionamiento de la red. La participación en el PFPD es un factor que las profesoras consideran clave para formar parte, posteriormente, de la red. Puesto que el programa tiene unos límites en tiempo y espacios de trabajo, se plantea que es preciso vislumbrar otros ambientes académicos que posibiliten continuar explorando los temas estudiados en los proyectos que realizaron en el marco del PFPD u otros asuntos de su interés. Como puede leerse en la siguiente cita, el programa les permitió trabajar en grupo y les dio bases para realizar investigaciones. En consecuencia, se asocia el trabajo en la red con una posibilidad para seguir avanzando.

Debemos contar con que los docentes de inglés (todos) hagan el PFPD para que así todos podamos contribuir con los aportes y apuntemos a un mismo objetivo. (Cuestionario, Alondra)

Por otra parte, las participantes también están de acuerdo en que gracias a la labor de dar a conocer la naturaleza y el funcionamiento de una red de docentes se hicieron conscientes de sus beneficios. En las siguientes evidencias, encontramos que las profesoras resaltan la labor que se hace para la divulgación y contacto de los miembros de la red, los temas tratados oportunamente de acuerdo con lo acordado con los asistentes a los encuentros, la puntualidad y los esfuerzos por mantener un trabajo adecuado.

Uno ve que es un trabajo muy juicioso, o sea que es algo muy, muy responsable, muy organizado.... Entonces eso como que lo lleva a uno a venir siempre. (Entrevista 2, Cristina)

Que lo tengan en cuenta a uno... eso es importante para que uno se motive, se motive a continuar. (Entrevista 2, Catalina).

Llama la atención que, si bien las profesoras contaban con acceso a Internet, las convocatorias para las sesiones de encuentro de la red o para recordarles al respecto, no siempre tenían el efecto esperado. En su mayoría, dependían de la iniciativa de la Universidad para compartir materiales de interés o para citarlas. Esto último era más efectivo si se realizaba por vía telefónica, como se puede leer en el siguiente apartado.

Pero síi me parece bien importante la comunicación telefónica. Sí... O sea, esa es como clave porque cuando tú hablas por teléfono tienes como la posibilidad de que la otra persona no se atreva a decirte no, yo no puedo (Entrevista 2, Catalina).

Finalmente, merece destacarse que además de velar por la organización y ambiente de trabajo en los encuentros de la red, se reconoce que la Universidad brinda acceso a bibliotecas especializadas que serán útiles en las investigaciones de los docentes. A su vez, esto crea hábitos en el uso de herramientas como Internet y en la búsqueda de materiales que apoyen su labor docente.

\section{Apoyo externo}

En esta tercera categoría informamos acerca del rol de las instituciones educativas y organismos gubernamentales en el trabajo en red de los docentes de inglés participantes en nuestro estudio y pertenecientes a instituciones de la ciudad de Bogotá.

El apoyo que los miembros de la red necesitan empieza por los mismos colegios en donde laboran. Primero, las profesoras se quejan de la falta de compromiso de sus compañeros de trabajo hacia el crecimiento profesional. Según ellas esto se debe, por 
un lado, a que son profesores que llevan ejerciendo muchos años y no tienen otro interés que el de cumplir con las obligaciones que la jornada de trabajo les exige. Por otro lado se encuentran los profesores temporales, quienes pueden estar interesados, pero el tiempo no les permite emprender programas de desarrollo profesional ni involucrarse en proyectos con sus compañeros. Como expresa una docente,

Para poder hacer esto debe haber más profesores comprometidos en el área de inglés, ya que en este momento estoy sola y las personas que enseñan inglés conmigo no están tan interesadas en hacer y desarrollar el proyecto. Cuando las invité a venir conmigo al PFPD no quisieron (Cuestionario, Alondra).

Mis compañeros no manifiestan interés por lo que les dije. Ellos no vienen en propiedad [no son docentes con contrato fijo]. Ellos están dos meses, un mes se van. Esa es una de las dificultades. (Entrevista 1, Alondra)

En segundo lugar, pero no menos importante, se encuentra la necesidad del apoyo de las directivas de los colegios en el trabajo en red. Este apoyo se puede traducir en divulgación y espacio. Esto tiene que ver con una oportunidad oficial para socializar las acciones del docente en la red y la posibilidad de aplicar sus proyectos a nivel institucional. Para las socializaciones, los docentes piden espacio en las reuniones de área y espacio para compartir con otras jornadas, pues como indica una profesora, «no se da el tiempo requerido para este tipo de ejercicio. $\mathrm{Y}$ es difícil que los profesores brinden tiempo extra para este trabajo» (Cuestionario, Cristina). En opinión de otra docente, es preciso "permitir que en las reuniones de área se pueda dedicar el espacio para compartir y en las semanas de evaluación institucional, compartir con las otras jornadas» (Cuestionario, Mariana).

Se plantea que para lograr el apoyo institucional, el papel de la Secretaría de Educación de Bogotá debe ser totalmente activo en cuanto a divulgación, por cuanto «sí tienen el poder de convocatoria, si llega un comunicado, hay un encuentro; inicialmen- te sería en las localidades, pero podría extenderse» (Entrevista 1, Catalina).

Las profesoras también manifiestan que se requiere un apoyo de la Secretaría de Educación en cuanto a recursos, pues en sus instituciones son bastante limitados. Resaltan que es necesario contar con el material suficiente para poder ejecutar cada una de las etapas de los proyectos y no detenerse por falta de recursos económicos. Los siguientes testimonios así lo indican:

Pues cuando uno tiene una idea de hacer cosas nuevas... pues ellos [dicen] sí, adelante, pero cuando uno necesita por ejemplo fotocopias, pues dirían 'no, no hay, porque el presupuesto es escaso'. (Entrevista 1, Cristina)

Apoyo económico. Lograr que sea una política institucional - local. (Cuestionario, Adriana)

El papel de la Secretaría de Educación de Bogotá no debe quedarse en la financiación de PFPD. También es importante «proveer de información, capacitación y además generar los espacios para enterar a los docentes de inglés de la localidad de este proyecto» (Cuestionario, Catalina). Se plantea la necesidad de que existan capacitaciones, en cuanto al uso de herramientas, que permitan el trabajo en red. Una de ellas es Internet, que sorprendentemente, en la era de la tecnología, es uno de los obstáculos con los que se enfrenta la red, pues el no uso de la informática impidió una mayor fluidez. Los docentes no están capacitados para aprovechar esta herramienta al máximo y, en su mayoría, desconocen las múltiples opciones comunicativas que ofrece.

Pues sería ponernos a comunicarnos más por la parte de, de Internet... hacer más útiles esas herramientas... como apropiarnos más de esa parte ¿no?... Tener un contacto más directo. De pronto un día que podamos chatear, que de pronto haya una video conferencia, algo así. Que así no estemos aquí presentes en tiempo físico, sí estemos como todos conectados. Y también puede ser una experiencia diferente porque por lo menos yo no sé chatear, entonces eso sería algo para aprender y que lo obligaría a uno a ver otras perspectivas de la situación. (Entrevista 2, Adriana) 
De acuerdo con lo presentado en este apartado, la base del trabajo en red es la autonomía de los docentes representada en el compromiso y responsabilidad con la que ejercen su función como miembros de la misma. Asimismo, la guía de la institución formadora, que permite su funcionamiento de una manera ordenada y armónica. Por otro lado, el gran impedimento que ha tenido la fluidez de la red es la falta de apoyo externo, a saber: de la institución educativa a la que pertenecen, sus directivas y compañeros y de la Secretaría de Educación Distrital con recursos, divulgación y capacitación.

\section{Conclusiones e implicaciones}

¿Qué favorece el trabajo en red de profesores de inglés de Bogotá después de su participación en un programa de formación permanente?

Yo creo que una red. La idea de una red de profesores de inglés es de personas que se reúnen y que tienen un interés en común. En el caso nuestro es el de mejorar el desempeño en la enseñanza del inglés y también el propio mejoramiento del inglés, y compartir experiencias para aplicarlas en el aula; también estar al tanto de la innovación, de cosas nuevas (Entrevista 2, Cristina).

Encontramos que el haber asistido al programa de formación permanente en el año 2006 fue el principal factor que permitió a las profesoras participantes en este estudio su vinculación en la red. Durante el PFPD, y específicamente en el componente de investigación e innovación, las profesoras despertaron su inquietud investigativa y con ello la necesidad de explorar otros campos en la educación no solamente a lo largo del mismo, sino después de culminarlo.

En segundo lugar, puesto que era requisito del programa que los profesores se reunieran en grupo para desarrollar un proyecto de investigación-acción e innovación, esta dinámica les reveló las ventajas de trabajar en grupo, así como la posibilidad de trabajar en temas de metodología y lengua inglesa, entre otros. Así, y como apuntan Bojacá (2001) y Calvo (2004), las docentes participantes en nuestro estudio corroboraron que el trabajo en comunidad posibilita la puesta en común, el apoyo mutuo y la reflexión sobre las realidades de su contexto escolar.

Se destacan otros dos factores que favorecen el trabajo en red de los docentes: la autonomía del profesorado y el papel de la institución formadora en el proceso. La autonomía de los docentes se percibe como la base de la red, ya que los impulsan la motivación y compromiso que tienen hacia su evolución personal y académica.

La evolución personal también da cuenta del interés en mejorar su nivel de lengua inglesa, herramienta vital para su quehacer docente. Observamos que lograron este cometido gracias a espacios previstos en las diferentes sesiones de la red, a la interacción con tutoras, asistentes de investigación e invitados extranjeros, quienes compartían sus conocimientos sobre cultura en talleres especializados para practicar el idioma. En este orden de ideas, constatamos que el concurso decidido de los docentes es un factor esencial para que se desarrolle una identidad personal a través del compromiso, la imaginación y la formación continua pues, como señala Brew (2006), todo esto hace posible la construcción de conocimiento.

La evolución académica que buscan, se refiere a dos aspectos de su interés: su práctica pedagógica y la actualización. El interés por mejorar la práctica pedagógica no es nada más que el reflejo de la responsabilidad y compromiso que sienten hacia sus estudiantes. Es por esto que en el momento de escoger los temas que desearían tratar en futuras sesiones salen a relucir los relacionados con la metodología de la lengua inglesa. Además, la investigación y posterior publicación de sus trabajos es para ellos una opción para trascender profesionalmente, enriquecerse a la vez con nuevos conocimientos y ayudar a sus estudiantes a tener una mejor práctica. La actualización hace referencia, en este caso, a la conexión con los eventos académicos de la Universidad. Estar al tanto de las conferencias, encuentros de investigación y otros acontecimientos relacionados con el área es una forma de explorar y descubrir nuevos avances en cuanto a educación, investigación y uso de la lengua. 
En relación con el papel de la institución formadora en el proceso de constitución y funcionamiento de la red, colegimos que su acompañamiento se ve reflejado en el ambiente de trabajo y en la guía que se ofrece a los docentes. Como equipo encargado de organizar los encuentros y animar la comunicación entre los participantes, observamos que fue muy importante crear un ambiente de trabajo agradable. La puntualidad en los eventos, la planeación oportuna de las actividades, el cumplimiento de lo prometido o acordado en los encuentros y el simple hecho de compartir unas onces como compañeros de red, fueron factores fundamentales para crear una armonía que, de alguna forma, motiva a los miembros a continuar, generando así una atmósfera diferente a la del colegio. En este punto cabe mencionar que la compatibilidad que existía entre los miembros contribuyó también al buen desarrollo de las actividades propuestas por los participantes en la red.

Finalmente, merece señalarse que el papel de la institución formadora no debe ser el de un estamento superior que dirige y controla todo, sino el de una guía que avanza conjuntamente con los docentes. Sentirse parte del mismo grupo en el que está la Universidad y saber que los tienen en cuenta, es muy importante para que los docentes se involucren y quieran sacar adelante los proyectos de la red a la que pertenecen o para avanzar hacia el logro de las metas personales que se han trazado. Como señala una docente, es importante «que lo tengan en cuenta a uno... eso es importante para que uno se motive a continuar» (Entrevista 2, Catalina).

¿Qué impide el trabajo en red?

La dinámica de la institución educativa es muy compleja y a veces hay tantos aspectos de toda índole para desarrollar en dos horas de reunión de área... Entonces lo esencial, lo importante, lo fundamental siempre se queda de último o nunca se toca (Entrevista 1, Mariana).

Debemos contar con que los docentes de inglés (todos) hagan el PFPD para que así podamos contribuir con los aportes y apuntemos a un mismo objetivo (Cuestionario, Alondra).
La falta de apoyo externo entorpece el trabajo en red. Los docentes señalan y reiteran en diversas ocasiones que no reciben el suficiente respaldo de sus colegios. Adicionalmente, sus compañeros de trabajo no demuestran interés por los proyectos y no están dispuestos a invertir tiempo en otras actividades. Esta falta de interés puede estar relacionada con el hecho de que los compañeros no asistieron al PFPD y, por lo tanto, no están familiarizados con el mundo de la investigación. Otra explicación puede radicar en asuntos relacionados con los tipos de vinculación del profesorado o la permanencia de los mismos en la carrera docente. Las participantes en nuestro estudio perciben que algunos profesores llevan muchos años ejerciendo y no están dispuestos a invertir tiempo adicional a la jornada escolar en trabajo extra. También aluden con preocupación al caso de profesores de inglés que no están nombrados de planta y deben rotar de colegio en colegio. Al respecto advierten que, si bien estos docentes están interesados en participar en eventos de desarrollo profesional, no cuentan con el tiempo requerido.

Por otra parte, llama la atención que las directivas de los colegios afirman estar interesadas en que sus docentes participen en espacios de actualización y formación permanente, pero no abren un espacio oficial para la socialización y la discusión del trabajo en red. Por ello, las participantes consideran necesario

Hacer un trabajo con las secretarías [de educación]... con los cadeles ${ }^{6}$ porque ellos sí tienen el poder de convocatoria. Entonces, si llega un comunicado, hay un encuentro... pues inicialmente sería como en las localidades, pero eso podría extenderse. (Entrevista 2, Cristina)

Apoyo económico... Lograr que sea una política institucional - local. (Cuestionario, Adriana)

Encontramos que para posibilitar la consolidación de una red, y así asegurar una estrategia que dé continuidad al trabajo iniciado en los PFPD, se

6 Instancias de carácter técnico, administrativo y pedagógico, a través de las cuales la Secretaria de Educación de Bogotá busca desconcentrar la administración del servicio educativo en cada una de las localidades de la ciudad. 
requiere que los entes gubernamentales -en nuestro caso la Secretaría de Educación de Bogotá- facilite los recursos necesarios para el funcionamiento de la red y la ejecución de proyectos que de allí surjan. Asimismo, es preciso que dicha entidad esté involucrada en la divulgación de la red, de manera que llegue a los profesores y colegios, tanto con sus mensajes de respaldo como con las respectivas convocatorias.

Por otra parte, es necesario involucrar o por lo menos concientizar a los directivos de las instituciones educativas de que, más que nadie, ellos y sus

\section{Bibliografía}

Álvarez, J. (2009). An exploration of Colombian EFL teachers' knowledge base through teachers' reflection. En: Revista Linguagem \& Ensino, 12 (1), 73-108.

Arias, E. (2008). Teachers' professional development through a study group. Universidad Distrital Francisco José de Caldas. Bogotá, Colombia. Thesis. Master in Applied Linguistics to TEFL.

Birchak, B., Connor, C., Crawford, K., Kahn, L., Kaser, S., Turner, S. y Short, K. (1998). Teacher study groups. Building community through dialogue and reflection. Urbana, IL: National Council of Teachers of English.

Bojacá, B. (2001). Redes nacionales para la transformación de la formación docente en didáctica de lengua materna. En: Memorias Segundo Congreso Colombiano de Lectoescritura en Lengua Materna y Lengua Extranjera. Bogotá: Universidad Distrital Francisco José de Caldas, 49-57.

Brew, A. (2006). Research and teaching. Beyond the divide. Nueva York: Palgrave Macmillan.

Brown, J. (2002). Understanding research in language learning: a teacher's guide to statistics and research design. Cambridge: Cambridge University Press.

Burns, A. (1999) Collaborative action research for English language teachers. Cambridge: Cambridge University Press.

Calvo, G. (2004). La formación de los docentes en Colombia. Estudio diagnóstico. Bogotá: Universidad Pedagógica Nacional-UNESCO-IESALC. instituciones se benefician de los proyectos de investigación que se desarrollan en el campo educativo. Así se podrá cristalizar el apoyo que las instituciones educativas prometen brindar a los docentes que se registran en los PFPD. Finalmente, una redefinición de los modelos de formación permanente de docentes que supere la tradición del entrenamiento para dar prelación al trabajo cooperativo entre la universidad y la escuela, establece las bases para que apoyemos el trabajo en redes e indaguemos aún más respecto a la manera como los docentes experimentan esta nueva relación con pares académicos.

Cárdenas, B. (2003). Teacher researchers as writers: a way to sharing findings. Colombian Applied Linguistics Journal, 5, 49-64.

Cárdenas, B. y Nieto, C. (2010). El trabajo en red de docentes de inglés. Bogotá: Universidad Nacional de Colombia.

Cárdenas, M. (2002). Teacher research as a means to create teachers' communities in inservice programs. HOW, A Colombian Journal for English Teachers. 9, $1-6$.

(2004). Las investigaciones de los docentes de inglés en un programa de formación permanente. Ikala, Revista de Lenguaje y Cultura, 9 (15), 105-137. (2006). PFPD red PROFILE-Red de profesores de inglés como lengua extranjera para el fortalecimiento de sus competencias comunicativa, pedagógica e investigativa en la educación básica y media. Bogotá: Universidad Nacional de Colombia, Departamento de Lenguas Extranjeras. Mimeo.

Cárdenas, M. y Nieto, M. (2007). El papel de la investigación-acción en el trabajo de grupos de estudio de docentes de inglés de educación básica y media del sector oficial. Simposio Internacional Investigación Acción y Educación en Contextos de Pobreza. Bogotá: Universidad de la Salle, mayo de 2007. Memorias CD.

Cárdenas, M., González, A. y Álvarez, J. (2010). El desarrollo profesional de los docentes de inglés en ejercicio: algunas consideraciones conceptuales para Colombia. En FOLIOS, 31, 49-67. 
Castellanos, A. (2009). The scope of professional development in a virtual study group. Universidad Distrital Francisco José de Caldas. Bogotá, Colombia. Thesis. Master in Applied Linguistics to TEFL.

Collay, M., Dunlap, D., Enloe, W. y Cagnon Jr, G. (1998). Learning circles, creating conditions for professional development. Thousand Oaks: Corwin Press, Sage.

Gómez, D. y Pineda, D. (2009). Learning to do research: the experience of the study group in autonomy and new technologies. En: M. L. Cárdenas Beltrán (ed.). Investigación en el aula en L1 y L2. Estudios, experiencias y reflexiones. Bogotá: Universidad Nacional de Colombia. Facultad de Ciencias Humanas. Biblioteca Abierta, 195-204.

González, A., Montoya, C. y Sierra, N. (2001). EFL teachers look at themselves: could they grow together? HOW. A Colombian Journal for Teachers of English, 9, 29-33.

(2002). What do EFL teachers seek in professional development programs? Voices from the teachers. En: Íkala, Revista de Lenguaje y Cultura. 7, 29-50.

Kirschner B., Dickinson R. y Blosser C. (1996). From cooperation to collaboration: the changing culture of a school/university partnership. En: Theory into practice, 35(3), 205-213

Malagón, L. y Cárdenas, M. (2007). La creación de grupos de estudio: vivencias en el marco de un programa de formación permanente de docentes de inglés. En Signum, 10 (1), 73-94.

Martínez, M. (2004). De redes sociales a redes pedagógicas: campo para constitución del maestro como sujeto político. Bogotá: Universidad Pedagógica Nacional.

McNulty, M. y Quinchía, D. (2007). Designing a holistic professional development program for elementary school English teachers in Colombia. En: PROFILE. Issues in Teachers' Professional Development, 8, 131-143.

Merriam, S. (1988). Case study research in education: a qualitative approach. San Francisco: Jossey-Bass.

Ministerio de Educación Nacional (2002). Decreto 1278 de Junio 19 de 2002. Bogotá: Presidencia de la República de Colombia.
Ministerio de Educación Nacional (2002). Decreto 2277 de Septiembre 1 de 1979. Bogotá: Presidencia de la República de Colombia.

Moreno, L. y Palencia, S. (2001). Teacher communities as a way to support professional Development. En HOW A Colombian Journal for English Teachers. Pasto. 9 50-56

Murphy, C. y Lick, D. (1998). Whole-faculty study groups. A powerful way to change schools and enhance learning. Thousand Oaks: Corwin Press.

Ochoa, L. (2004). Teacher Communities. Bogotá: Universidad Distrital. Tesis de Maestría en Lingüística Aplicada a la Enseñanza del Inglés.

Ochoa, M. (2005). Reflective ELT meetings of a public elementary school teachers' group. Bogotá: Universidad Distrital. Tesis de Maestría en Lingüística Aplicada a la Enseñanza del Inglés.

Oliphant, K. (1996). Teacher development groups: growth through cooperation. En Íkala, Revista de Lenguaje $y$ Cultura, 1 (2), 67-86.

Secretaría de Educación de Bogotá D. C. (2006). Plan territorial de formación docente 2006-2007. Una oportunidad de transformación Pedagógica de la escuela. Bogotá: Serie Lineamientos de Política Alcaldía Mayor de Bogotá.

Sierra, A. (2007a). Developing knowledge, skills and attitudes through a study group: a study on teachers' professional development. En Íkala, Revista de lenguaje y cultura, 12 (18), 279-305.

(2007b). The professional development of a facilitator through a study group. En: PROFILE. Issues in Teachers' Professional Development, 8, 91-101.

Strauss, A., y Corbin, J. (1990). Basis of qualitative research. Grounded theory procedures and techniques. Newbury Park: Sage Publications.

Torres G. e Isaza, L. (2000). Evaluación de los Programas de Formación Permanente de Docentes en Bogotá entre 1996 y 2000. IDEO.

Vergara, O., Hernández, F. y Cárdenas, R. (2009). Classroom research and professional development. En: PROFILE, Issues in Teachers' Professional Development, 11, 169-191. 
